Ann. Biol. anim. Bioch. Biophys., 1979, 19 (3 B), 881-888.

\title{
Importance de la voie vagale sensitive : recherche d'une technique de déafférentation sélective chez le porc et le mouton
}

\author{
par Béatrice DARCY, M. FALEMPIN *, J. P. LAPLACE, J. P. ROUSSEAU * \\ avec la collaboration technique de C. GERMAIN, A. ROUSSEAU * et P. VILLIERS \\ Laboratoire de Physiologie de la Nutrition, I.N.R.A. \\ 78350 Jouy-en-Josas, France. \\ * Laboratoire de Neurophysiologie Végétative, SN4. \\ Université de Lille 1, B. P. 36, 59650 Villeneuve d'Ascq, France
}

Summary. The role of vagal afferences : development of a deafferentation technique in pigs and sheep.

The vagus nerve is composed of efferent motor fibers, whose cell bodies are located in the brain stem, and of many afferent sensory fibers whose neuronic somas are located in the nodose ganglion. The present work deals with the surgical selective suppression of sensory pathways (deafferentation) in pigs and sheep. On its cervical route, the vagus nerve in pigs shows a definite anatomical separation between its motor and sensory pathways, sufficiently large to permit the efferent fiber bundle and the nodose ganglion, containing the sensory cells, to be surgically dissociated. The integrity of digestive motility after unilateral truncular vagotomy was checked, as well as the absence of cardio-respiratory or digestive abnormality after the removal of only one nodose ganglion. Bilateral removal of the nodose ganglions caused the animals to die within 2 to 4 days. Bilateral suppression of afferences originating below the diaphram was obtained in two steps : 1) removal of the nodose ganglion on the left side by a retroparotidian route ; 2 ) truncal vagotomy on the right side at the level of the diaphragm after thoracotomy. The vagal efferences on the left side could thus be retained, while the afferences originating below the diaphragm on either side were interrupted. This deafferentation was compatible with a prolonged survival. It eliminated the differences due to the effects of the physico-chemical characteristics of the meal on antral motility. The problem in sheep was more complex :1) motor nerve bundles were not always clearly individualized at the surface of the nodose ganglion ; thus, when stimulating the contralateral vagus nerve, effective unilateral deafferentation had to be confirmed by the absence of the classically described gastric reflex contraction of long latency (Dussardier, 1960) ; 2) the anatomical arrangement of the thoracic course of the vagus nerves was different, and section of the dorsal trunk above the diaphragm immediately arrested forestomach motility. With the procedure used in pigs, we failed to obtain sheep completely deprived of digestive afferences. Contrary to this finding, the exeresis of only one nodose ganglion was followed by a transitory arrest of the forestomach cycle, ranging from some minutes to several hours, and in one case by even a definitive arrest. After the meal, gastric contraction frequency was lower and less durable than in intact animals. Two out of 8 animals died from pulmonary odema within 12 to $33 \mathrm{hrs}$, while the section of only one cervical vagus nerve was well tolerated. 
L'intensité et la chronologie des phénomènes moteurs gastro-intestinaux sont fonction des caractéristiques physicochimiques de l'aliment. Cet ajustement de la fonction motrice est pour partie le résultat de l'information du système nerveux central par voie humorale, du fait des variations du milieu intérieur, ef par voie nerveuse grâce aux afférences provenant des divers types de récepteurs présents dans la paroi du tube digestif. L'existence de ces récepteurs est fondée sur la mise en évidence des modifications de la motricité induites par des stimulations endoluminales du tractus (voir sur ce point les revues de Hunt et Knox, 1968, et de Thomas et Baldwin, 1968) et sur l'analyse électrophysiologique (lggo, 1955, 1957 ; Leek, 1969 ; Mei, 1970). Ces récepteurs sont le point de départ de réflexes digestifs qui rendent compte par exemple des effets liés au volume du repas (Hunt et MacDonald, 1954) : la distension de l'estomac stimule l'évacuation gastrique ; la réplétion intestinale, notamment par des contenus à forte teneur en matière sèche, l'inhibe comme cela est vérifié chez le Porc (voir revue de Laplace, 1975). L'accélération de la motricité réticulaire chez le Mouton peut également être suscitée par la mise en jeu de tels réflexes à partir d'afférences bucco-pharyngées ou prenant origine au niveau des zones réflexogènes du réticulo-rumen (Titchen, 1958 ; Comline et Titchen, 1961 ; Ruckebusch et Laplace, 1967). D'une façon générale, les réflexes en cause peuvent emprunter diverses voies. Ils sont selon les cas dits courts (intramuraux) ou longs lorsque l'information se projette au niveau du système nerveux central. Certains des réflexes longs empruntent la voie du nerf pneumogastrique (ou vague).

Le vague est un nerf mixte comportant des fibres motrices (efférentes) dont les corps cellulaires sont situés dans le bulbe rachidien (nucleus parasympathicus nervi vagi), et des fibres sensitives (afférentes) dont les somas neuroniques sont localisés dans le ganglion plexiforme (ganglion distale nervi vagi). L'importance relative des contingents moteur et sensitif du nerf vague est bien précisée chez le Mouton (Dussardier, 1960). Tel n'est pas le cas chez le Porc. Cette espèce présente pourtant, à l'instar du Chat (Mei, 1966), l'intéressante particularité anatomique d'une séparation macroscopique des deux contingents au niveau du ganglion plexiforme. Parallèlement à l'étude de l'importance anatomique de la voie sensitive (travail en cours), il nous paraît intéressant de rechercher une technique de déafférentation vagale sélective adaptée au Porc et au Mouton, pour disposer de préparations permettant d'apprécier l'importance fonctionnelle de la voie vagale sensitive.

\section{A. - Cas du Porc}

1. Vérifications anatomo-histologiques. - La séparation macroscopique des voies motrice et sensitive est initialement vérifiée par dissection fine chez 4 Porcs. Elle est effective sur un bref trajet $(10$ à $15 \mathrm{~mm}$ ) du vague cervical au niveau du ganglion plexiforme (fig. 1). Cette séparation par un plan conjonctif est suffisante pour autoriser la dissociation chirurgicale du faisceau de fibres motrices d'une part et du paquet ganglionnaire d'autre part, aussi bien chez le porcelet de $30 \mathrm{~kg}$ (12 animaux) que chez des porcs de 60 à $70 \mathrm{~kg}$ ( 6 animaux). L'authenticité du ganglion plexiforme est vérifiée par la seule présence, dans l'amas dissocié chirurgicalement, des corps cellulaires des neurones sensitifs (fig. 2). 
2. Contraintes fonctionnelles. - Le contingent vagal afférent véhicule tout à la fois l'intéroception viscérale cardiopulmonaire et digestive. L'ablation chirurgicale bilatérale des ganglions plexiformes est responsable d'altérations graves de la fonction cardio-respiratoire conduisant à la mort à brève échéance. Nous constatons ce phénomène chez 2 porcs, morts en 2 à 4 jours par œdème pulmonaire aigu. Compte tenu de l'objectif recherché, il est vérifié que la vagotomie tronculaire unilatérale droite (2 porcs) ou gauche (2 porcs) ne perturbe pas l'activité électromyographique gastrique ; la vagotomie tronculaire bilatérale au niveau diaphragmatique ( 2 porcs) conduit à un retard cumulatif de l'évacuation gastrique avec distension considérable de l'estomac, et

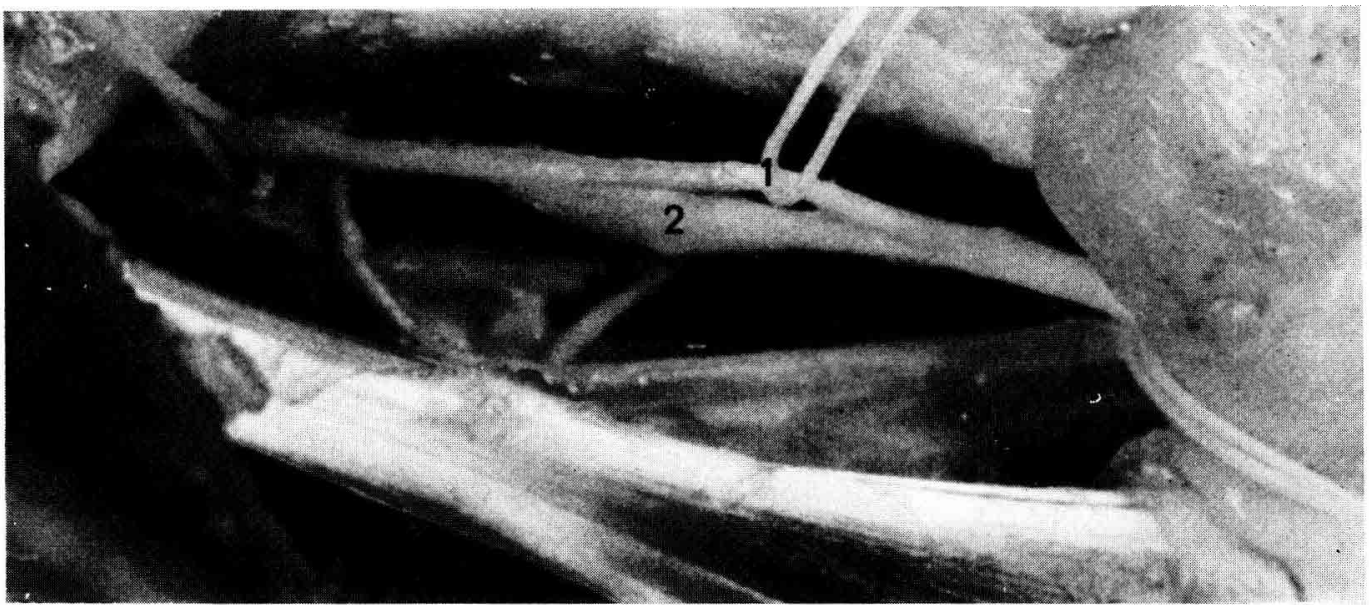

FIG. 1. - Mise en évidence macroscopique de la séparation anatomique entre la branche efférente (1) ef la branche offérente sensitive (2) du vague cervical gauche chez le Porc. La branche sensitive est caractérisée par la présence d'un renflement: ie ganglion plexiforme. L'extrémité céphalique de la préparation est située à gauche sur la photo.
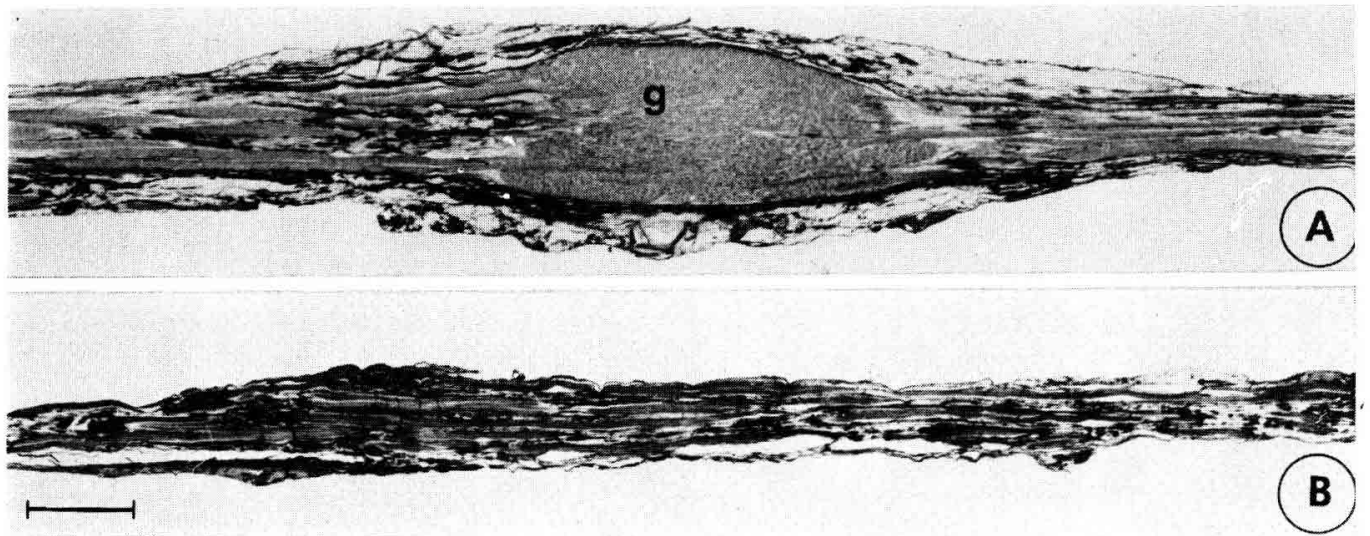

FIG. 2. - Coupes longitudinales des deux branches du vague cervical, après séparafion chirurgicale. On observe en A la masse des cellules du ganglion plexiforme $(\mathrm{g})$, confirmant la nature afférente de cette branche vagale. Les corps cellulaires sont absents en $B$, au niveau de la branche efférente. Etalonnage : 1,5 mm. 
éventuellement à la mort de l'animal ; l'ablation chirurgicale unilatérale d'un ganglion plexiforme ( 2 porcs) n'est responsable d'aucune difficulté cardiorespiratoire ou digestive cliniquement décelable.

3. Principe de la technique chirurgicale. - Sur la base des éléments ci-dessus, la technique chirurgicale de déafférentation vagale sélective proposée comporte deux étapes, en une même intervention sous anesthésie générale.

- Exérèse du ganglion plexiforme gauche, abordé par voie rétroparotidienne ; la voie sensitive est donc supprimée du côté gauche, mais la voie efférente motrice est préservée.

- Vagolomie tronculaire droite au niveau du diaphragme, à la faveur d'une thoracotomie. Les afférences d'origine post-diaphragmatique, empruntant l'un ou l'autre des 2 vagues sont supprimées ; la voie motrice droite est également interrompue.

Cette intervention est réalisée avec succès sur une vingtaine de porcs. L'intervention homologue contralatérale n'est pas jusqu'ici mise en œuvre.

4. Limites actuelles de la technique. - Au tolal, les porcs ainsi préparés sont privés d'intéroception vagale d'origine post-diaphragmatique, mais conservent intactes la voie motrice gauche et l'intéroception vagale d'origine cardio-pulmonaire du côté droit. Cependant, cette déafférentation ne peut être considérée comme totale qu'en l'absence de rameaux de communication entre le trone vagal droit et son homologue gauche en amont du diaphragme. Or la dissection complète des pneumogastriques met en évidence de tels rameaux sur leur trajet intra-thoracique, entre les plexus cardiopulmonaires et le diaphragme. Le nombre et la taille de ces rameaux, comme leur disposition exacte, sont relativement variables d'un porc à l'autre. La nature des fibres ainsi échangées entre les 2 vagues n'est pas connue ef l'évaluation de la signification fonctionnelle de ces rameaux est en cours.

En conséquence, la déafférentation réalisée comme il est décrit ci-dessus ne peut être considérée comme totale. Bien que la section des rameaux soit aisément réalisable, il paraît nécessaire d'en contrôler les effets particuliers éventuels avant que d'ajouter cette troisième étape à la technique chirurgicale proposée.
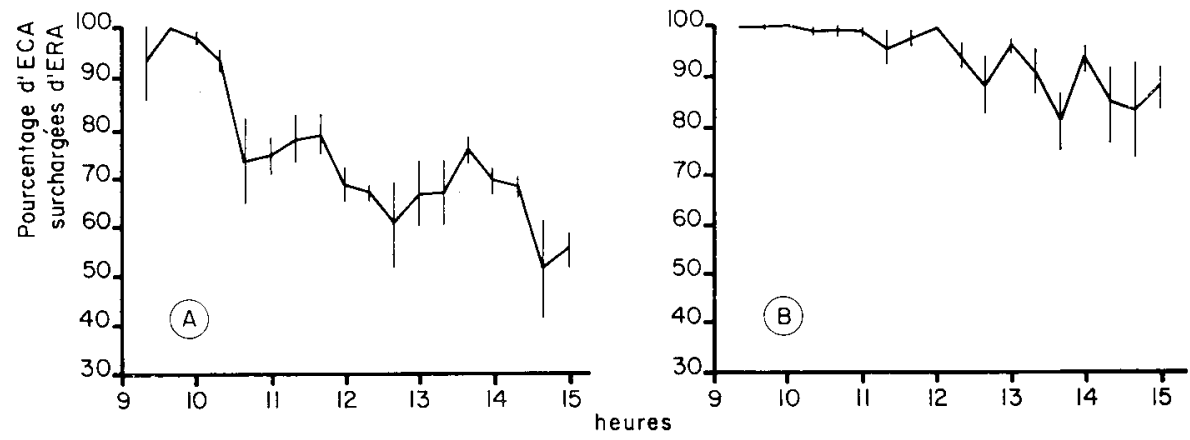

FIG. 3. - Evolution au cours des 6 h post-prandiales du pourcentage moyen (par période de $20 \mathrm{mn}$ ) de surcharge des activités de contrôle (ECA) par des activités de réponse (ERA), au niveau de l'antre gastrique chez le Porc, après ingestion de $960 \mathrm{~g}$ de matière sèche (aliment standard). Chaque graphique correspond à la moyenne de 6 épreuves. A - avant vagotomie sensitive ; B - après vagotomie sensitive. 
5. Premières observations sur la motricité antrale après déafférentation bilatérale. L'électromyogramme de l'antre gastrique après déafférentation vagale bilatérale est caractérisé par une très importante augmentation de la fréquence d'association d'une activité de réponse * à l'activité électrique rythmique de contrôle*. Chez le porc intact, dans l'heure qui suit l'ingestion d'un repas d'aliment standard, 100 p. 100 des activités de contrôle sont suivies d'une activité de réponse mais ce pourcentage décroît progressivement jusqu'à une valeur d'environ 50 p. 100 au bouł de 6 h (fig. 3A). Chez le porc déafférenté, on observe la persistance d'une activité maximale : la proportion d'activités de contrôle suivies d'une forte activité de réponse reste encore proche de 90 p. 100, $6 \mathrm{~h}$ après le repas (fig. 3B).

Des effets analogues peuvent être observés à la suite de l'ingestion d'aliment semisynthétique à base d'amidon de maïs purifié, ou de saccharose : l'inhibition de l'activité gastrique (faible pourcentage d'activité de réponse) disparaît chez le porc déafférenté au profit d'une activité antrale importante quoique moins régulière que lors d'ingestion d'aliment standard.

En somme tout se passe comme si, globalement il y avait défaut de freinage de l'activité gastrique. Si l'on rapproche ce phénomène du fait que cette activité est essentiellement soumise au contrôle inhibiteur des réflexes entérogastriques d'origine duodénale, tout conduit à penser que ceux-ci empruntent, au moins pour partie, la voie vagale. Les modifications observées de l'activité antrale postprandiale témoigneraient alors de la réalité de la déafférentation.

\section{B. - Cas du Mouton}

Le cycle moteur du réticulo-rumen des polygastriques ruminants, tels que le mouton, est étroiłement contrôlé par les influx moteurs vagaux d'origine bulbaire. Le rythme de la motricité est nettement sous la dépendance des afférences d'origine périphérique. Le problème est donc de savoir l'importance des influx sensitifs vagaux dans l'élaboration du rythme gastrique.

1. Aspects anatomo-fonctionnels ef techniques. - Au plan chirurgical, le problème est plus complexe chez le Mouton que chez le Porc, pour deux raisons anatomiques. La première est que la séparation au niveau de l'émergence du vague, entre le contingent moteur ef la masse des cellules sensitives du ganglion plexiforme est beaucoup moins nette chez le Mouton, comme cela a été observé antérieurement (Laplace, 1968). Il en résulte la nécessité de vérifier chez le Mouton la réalité de la déafférentation unilatérale, première étape du protocole. Cette vérification est basée sur le fait que la stimulation du bout central d'un nerf vague sectionné provoque par voie réflexe une contraction de longue latence du réseau-rumen (Dussardier, 1960). Lorsque nous portons une stimulation itérative sur le vague intact, contralatéral à la déafférentation, la réponse motrice directe, de latence inférieure à une seconde, provoquée par l'excitation des efférences vagales est suivie 4 à 12 s plus tard de la contraction réflexe, déclen-

(*) La terminologie utilisée pour désigner les composantes de l'activité électrique de l'antre gastrique est celle retenue par Laplace et Roman (1979) comme la moins susceptible d'induire des confusions quant à leur nature. 
chée par la stimulation centripète du contingent afférent du nerf (fig. 4A). Lorsqu'au contraire, le vague présumé déafférenté est stimulé dans les mêmes conditions, l'absence de la réponse réflexe doit en première analyse traduire la réalité de la déafférentation (fig. 4B).

A

$1 \mathrm{~s}$.

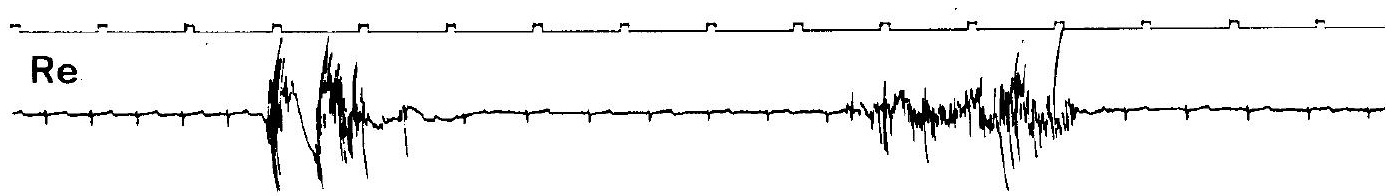

B

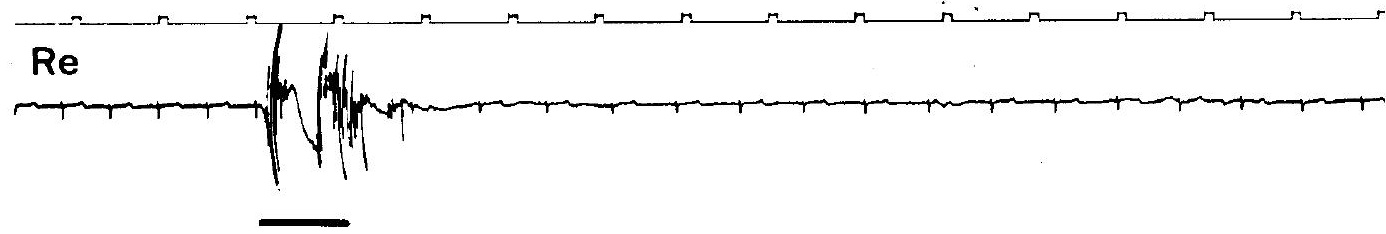

FIG. 4. - Vérification de la réalité de la déafférentation unilatérale chez le Mouton.

En A, la stimulation (trait) du vague normal contralatéral à la déafférentation déclenche une contraction directe du réseau $(\mathrm{Re})$, de faible latence, suivie d'une contraction réflexe due à l'activation des afférences.

En $B$, la stimulation (trait) du vague présumé déafférenté provoque seulement la contraction directe. L'absence de contraction réflexe témoigne du défaut d'afférences au sein du nerf stimulé.

La seconde raison est la conséquence de la disposition anatomique des vagues dans leur trajet thoracique. Chaque nerf droit et gauche donne une branche inférieure et une branche supérieure. Les deux branches inférieures fusionnent en un tronc œsophagien ventral, les deux supérieures en un tronc œsophagien dorsal. Or, la section de ce dernier, dans sa portion prédiaphragmatique, détermine un arrêt de la motricité (Duncan, 1953). La seconde étape du protocole ne peut donc pas être envisagée comme chez le Porc. Il faut sectionner d'un seul côté les deux branches inférieure el supérieure d'un même vague avant leur jonction avec leurs homologues contralatérales. En outre, l'émergence de filets cardiopulmonaires, qui intervient après la subdivision du vague en ses deux branches, interdit une section en amont de cette subdivision. La difficulté est d'ordre chirurgical : si la section de la branche supérieure d'un vague droit ou gauche est facile, celle de la branche inférieure, d'accès malaisé, n'est pas réalisée jusqu'ici de façon satisfaisante et certaine. II résulte de ces difficultés que nos premiers résultats concernent seulement les effets de la déafférentation unilatérale.

2. Conséquences de la déafférentation unilatérale. - La déafférentation unilatérale a des effets sur la motricité rumino-réticulaire ; elle peut éventuellement conduire à la mort de l'animal ( 2 moutons). La déafférentation unilatérale ( 9 moutons) est suivie d'un arrêt des contractions gastriques, qui persiste de quelques minutes à plusieurs heures. La récupération du rythme normal est obtenue en $60 \mathrm{~min}$ à $72 \mathrm{~h}$ selon les cas. 
Chez un animal, l'arrêt de la motricité est même définitif. Nous notons en plus chez deux moutons des troubles de la motricité de l'œsophage thoracique.

Pour évaluer l'influence de la réduction du volume des afférences post-diaphragmatiques, nous utilisons le test de la prise alimentaire. Chez l'animal normal, le repas déclenche immédiatement l'accélération du rythme gastrique (243 p. $100 \pm 122$ p. 100 du rythme préprandial). Celle-ci persiste au moins 30 min après l'arrêt d'un repas de $100 \mathrm{~g}$ de granulés ingérés en 3 à $4 \mathrm{~min}$ (fig. 5). Après déafférentation unilatérale, l'accélération pendant le repas n'est pas significativement inférieure (200 p. $100 \pm 40$ p. 100) ; la fréquence des contractions après le repas est par contre significativement moins importante ef l'accélération moins durable, puisque le retour au ryth me normal est réalisé dans les 5 min qui suivent la fin du repas (fig. 5).

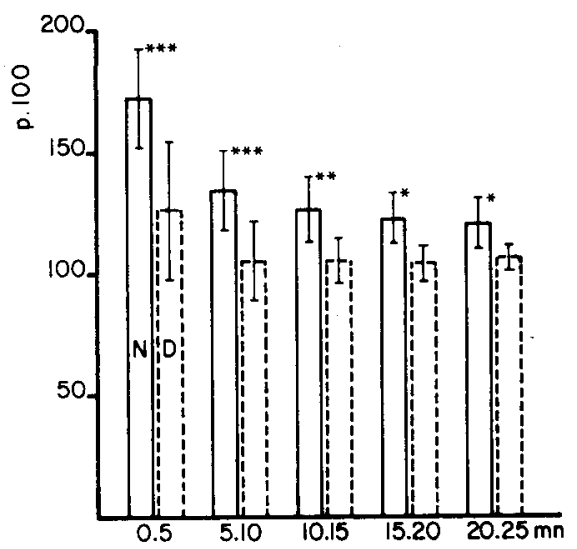

FIG. 5. - Comparaison des rythmes de contractions gastriques dans les minutes (0-5, 5-10, 10-15, 15-20, 20-25) qui suivent l'arrêf de la prise de nourrifure, entre l'animal normal (N) et l'animal ayant subi une déafférentation unilatérale (D). La fréquence moyenne des contractions gastriques, portée en ordonnée est exprimée en pourcentage de la fréquence préprandiale. Au delà de 5 min après la fin du repas, le rythme est à nouveau identique à un rythme préprandial chez les animaux déafférentés (moyennes de 6 animaux).

Différences, normal vs. déafférenté, testées, par le $\dagger$ de Student: ${ }^{* * *} p<0,001 ; * * p<0,01$; $* p<0,05$.

En conclusion, la déafférentation bilatérale est réalisable techniquement chez le Pörc ; elle pose encore des problèmes chirurgicaux chez le Mouton. La réduction des afférences vagales d'origine post-diaphragmatique, subtotale chez le Porc, partielle chez le Mouton, entraîne des modifications des réponses motrices gastriques à la prise alimentaire.

Commission CNERNA Digestion-Absorption/Association des Physiologistes, Paris 5-6 octobre 1978.

Remerciements. - Ce travail a bénéficié des aides n 77.7 .0462 et 77.7 .0463 de la DGRST, dans le cadre de l'action sur programme «Interrelations aliments-tube digestif $)$. 


\section{Références}

COMLINE R. S., TITCHEN D. A., 1961. Nervous control of the ruminant stomach, 10-22. In LEWIS D. Digestive physiology and nutrition of the ruminant, Butterworths. Londres.

DUNCAN D. L., 1953. The effects of vagotomy and splanchnotomy on gastric motility in the sheep J. Physiol. Lond., 119, 157-169.

DUSSARDIER M., 1960. Recherches sur le contrôle bulbaire de la motricité gastrique. Thèse Doct. ès Sci., Paris, 199 pp.

HUNT J. N., MACDONALD I., 1954. The influence of volume on gastric emptying. J. Physiol. Lond., 126, 459-474.

HUNT J. N., KNOX M. T., 1968. Regulation of gastric emptying, 1917-1935. In Handbook of Physiology, sect. 6 : Alimentary canal, vol. 4, Amer. Physiol. Soc. Washington.

IGGO A., 1955. Tension receptors in the stomach and the urinary bladder. J. Physiol. Lond., 128, 593-607.

IGGO A., 1957. Gastric mucosal chemoreceptors with vagal afferent fibres in the cat. Quart. exp. Physiol., 42, 398-409.

LAPLACE J. P., 1968. Sur les phénomènes mécaniques ef électriques du tractus digestif chez le mouton. Thèse Doct. $3^{\mathrm{e}}$ cycle (Physiologie animale), Lyon, 58-59.

LAPLACE J. P., 1975. Le transit digestif chez les monogastriques : 2. Phénomènes moteurs et mouvements des digesta. Ann. Zootech., 24, 489-551.

LAPLACE J. P., ROMAN C., 1979. Activités de la musculature gastro-intestinale et mouvements des contenus digestifs. Ann. Biol. anim. Bioch. Biophys., 19, 849-879.

LEEK B. F., 1969. Reticulo-ruminal mechanoreceptors in sheep. J. Physiol. Lond., 202, 585-609.

MEI N., 1966. Existence d'une séparation anatomique des fibres vagales efférentes et afférentes au niveau du ganglion plexiforme du chat. J. Physiol. Paris, 58, 253-254.

MEI N., 1970. Mécanorécepteurs vagaux digestifs chez le Chat. Exp. Brain Res., 11, 502-514.

RUCKEBUSCH Y., LAPLACE J. P., 1967. Repas fictif et pseudo-rumination chez le mouton après œesophagostomie. C. R. Soc. Biol., 161, 354-359.

THOMAS J. E., BALDWIN M. V., 1968. Pathways and mechanisms of regulation of gastric motility, 1937-1968. In Handbook of Physiology, sect. 6 : Alimentary canal, vol. 4, Amer. Physiol. Soc., Washington.

TITCHEN D. A., 1958. Reflex stimulation and inhibition of reticulum contractions in the ruminant stomach. J. Physiol. Lond., 141, 1-21. 